\title{
Regulation of Axonal Transport by Protein Kinases
}

\author{
Katherine Gibbs, Linda Greensmith and Giampietro Schiavo ${ }^{\ddagger}$
}

Sobell Department of Motor Neuroscience \& Movement Disorders, UCL Institute of Neurology, University College London, WC1N 3BG London, UK

†To whom correspondence should be addressed:

Prof. Giampietro Schiavo, Sobell Department of Motor Neuroscience \& Movement Disorders, UCL Institute of Neurology, University College London, Queen Square, WC1N 3BG London, UK Phone: ++44 203448 4334, Fax: ++44 207813 3107; e-mail: giampietro.schiavo@ucl.ac.uk

Words: 3,829

Key words: axonal transport, kinesin, cytoplasmic dynein, protein kinases, neurodegenerative diseases 


\section{Abstract}

The intracellular transport of organelles, proteins, lipids and RNA along the axon is essential for neuronal function and survival. This process, called axonal transport, is mediated by two classes of ATP-dependent motors, kinesins and cytoplasmic dynein, which carry their cargoes along microtubule tracks. Protein kinases regulate axonal transport through direct phosphorylation of motors, adapter proteins and cargoes, and indirectly, through modification of the microtubule network. The misregulation of axonal transport by protein kinases has been implicated in the pathogenesis of several nervous system disorders. Here, we review the role of protein kinases acting directly on axonal transport and discuss how their deregulation affects neuronal function, paving the way for the exploitation of these enzymes as novel drug targets.

\section{Axonal transport in neuronal function and survival}

Neurons are highly polarized cells, with axons that can reach over a meter away from the cell body in large mammals. This unique morphology makes neurons particularly dependent on active intracellular transport along the axon. Axonal transport is essential for the delivery of newly synthesized proteins, lipids and RNA, as well as organelles, to growth cones and synapses. It is also required for the removal of damaged cellular components and organelles for degradation and recycling. Furthermore, axonal transport has been shown to play a key role in long-distance communication between the distal region of the axon and the cell body, including neurotrophic and injury response signaling [1-3] and the regulation of axonal length [4]. Congruent with the crucial role of axonal transport in neuronal homeostasis, disruptions in axonal transport have been linked to neuronal dysfunction and degeneration $[5,6]$.

The complex spatial and temporal regulation of axonal transport is essential for controlled delivery of selected cargoes (see Glossary) to specific neuronal domains. Whilst a complete understanding of axonal transport 
regulation is still lacking, it has been known for some time that phosphorylation plays an important role in this process [7, 8]. However, deciphering which protein kinases are responsible for axonal transport modulation has been difficult, as kinase cascades display considerable crosstalk and play multiple roles in neuronal homeostasis [9-12]. Recently, significant progress has been made in dissecting out the role of individual kinases in this process and identifying their molecular targets.

Protein kinases are known to regulate axonal transport through direct phosphorylation of motors, adapter proteins and cargoes, and indirectly, through modification of the microtubule network. The diversity of protein kinases implicated in axonal transport regulation most likely underlies the complexity of this process and explains why transport characteristics are distinct between different types of neurons (for example, axonal transport is slower in sensory versus motor neurons) [13] and cargoes (for example, mitochondria move bidirectionally with frequent pauses, whilst signaling endosomes undergo sustained retrograde transport) [13, 14].

Here, we review the role of protein kinases that have been implicated in the direct regulation of axonal transport and discuss how kinase deregulation can affect neuronal function.

\section{The axonal transport machinery}

Axonal transport can be classified based on the speed at which cargoes move. Vesicles, organelles and RNA are transported by fast axonal transport at a speed of $50-200 \mathrm{~mm}$ per day $(0.5-3 \mu \mathrm{m} / \mathrm{s})$, whilst cytoskeletal components (such as neurofilaments and tubulin) and some soluble proteins are transported by slow axonal transport and move at rates of $0.1-3 \mathrm{~mm}$ per day [6].

Both types of transport are mediated by two classes of ATP-dependent motors - kinesin and cytoplasmic dynein - which carry their cargoes along microtubule tracks running the length of the axon [15]. Kinesin and dynein are 
assisted by adapter proteins, which link the motors to their cargoes and regulate the activity of the motors. Microtubules impart an intrinsic polarity to neurons, with their "plus" ends located at the axon tips and their "minus" ends within the cell body (Key Figure). This polarity confers directionality to axonal transport.

\section{Anterograde axonal transport}

Anterograde axonal transport refers to the movement of cargoes from the cell body towards the axon tips and is mediated by kinesin motor proteins.

Kinesin-1 motors are responsible for the fast anterograde axonal transport of most vesicles, organelles, proteins and RNA particles and the slow axonal transport of cytoskeletal proteins. Kinesin-1 family members are heterotetramers composed of two heavy chains (KIF5A-C) and two light chains (KLC1/2). KIFs comprise the motor domains and contain both ATP and microtubule binding motifs. KLCs interact with the tails of the KIFs and are thought to regulate cargo binding and motor activity [16] (see Box 1).

Kinesin-2 and kinesin-3 motors are also important for axonal transport. Members of the kinesin-2 family (KIF3A/KIF3B/KAP3) mediate the transport of late endosome-lysosomes [17, 18], whilst kinesin-3 motors (KIF1A and KIF1B $\beta$ ) carry synaptic vesicle precursors and dense core vesicles [19, 20].

\section{Retrograde axonal transport}

Retrograde axonal transport refers to the movement of cargoes from the axon tips to the cell body and is mediated by cytoplasmic dynein. Dynein is composed of two heavy chains and additional intermediate, light intermediate and light chains [15]. The heavy chain contains ATP and microtubule-binding motifs at its carboxy terminus, whilst the amino terminus interacts with the additional chains to form the cargo-binding domain. Dynein is found in a complex with dynactin [16], a multi-subunit cofactor that is essential for its function (see Box 1), and other adapter proteins, such as lissencephaly-1 (Lis1) and huntingtin (htt) [6]. 


\section{Kinases implicated in the regulation of axonal transport}

Protein kinases regulate axonal transport through direct phosphorylation of motors, adapters and cargoes (summarized in Table 1). Importantly, protein kinases also phosphorylate several factors involved in the regulation of microtubule stability, as discussed in detail elsewhere [21].

\section{Glycogen synthase kinase-3 beta (GSK3ß)}

GSK3 $\beta$ was originally identified as a regulator of glycogen metabolism [22], but has since been shown to be a multifunctional kinase with numerous roles in the nervous system [9]. In both invertebrate and rodent models, GSK3 $\beta$ acts as a negative regulator of anterograde and retrograde axonal transport (Figure 1 and 2) [23-28]. In Drosophila, overexpression of GSK3 inhibits the axonal transport of mitochondria [24, 25], amyloid precursor protein (APP)containing vesicles [27] and lipid droplets in larval segmental nerves [27]. There are conflicting views over whether GSK3 also inhibits the axonal transport of synaptic vesicles in these nerves [25, 27]. Stimulation of GSK3 $\beta$ by pathological proteins, such as presenilin 1 and amyloid beta $(A \beta)$ (see Relevance to CNS function and disease section) also inhibits bidirectional axonal transport in mammalian neurons [29, 30] (Table 1).

Pharmacological inhibition of GSK3 $\beta$ in hippocampal and sensory (DRG) neurons has been shown to stimulate the axonal transport of BDNFcontaining secretory vesicles, mitochondria and acidic organelles [24, 28]. Similar results are also seen in Drosophila larval segmental nerves carrying loss-of-function mutations in GSK3 [27]. However, in squid axoplasm, inhibition of GSK3 $\beta$ did not alter the axonal transport of membrane-bound organelles [26], indicating that this regulatory mechanism is not conserved between all species.

GSK3 $\beta$ regulates axonal transport through direct phosphorylation of motors and their adapters. In squid axoplasm, GSK3 $\beta$ was shown to phosphorylate KLC2, stimulating its release from cargoes, without influencing the microtubule binding or ATPase activity of the motor [26]. However, optical trap 
experiments in Drosophila indicate that GSK3 $\beta$ may instead act to reduce the number of active kinesins working at any one time, rather than the total number of motors bound to the cargo [27]. These contrasting results may represent differences in the regulation of axonal transport between species. GSK3 $\beta$ directly phosphorylates dynein intermediate chain 1B (DIC1B) and 2C (DIC2C), leading to inhibition of the retrograde axonal transport of acidic organelles [28]. GSK3 $\beta$-mediated phosphorylation of DIC1B was shown to reduce its interaction with Ndel1 and Lis1, which enhance dynein force production [31].

GSK3 $\beta$ has also been shown to regulate axonal transport through phosphorylation of motor adapter proteins. Collapsin response mediator protein-2 (CRMP-2) acts as a linker between kinesin-1 and specific cargoes such as tubulin heterodimers and TrkB-containing endosomes [32]. GSK3 $\beta$ mediated phosphorylation of CRMP-2 disrupts its association with KLC1 and is proposed to perturb the anterograde axonal transport of its cargoes [33] (Figure 1). However, this effect has yet to be demonstrated in live axonal transport assays.

Finally, GSK3 $\beta$ has been found to have differential effects on slow axonal transport, depending on the type of cargo being transported. For example, GSK3 $\beta$ directly phosphorylates tau [34], increasing its affinity for kinesin-1 and thereby accelerating its anterograde axonal transport [35]. By contrast, GSK3 $\beta$-mediated phosphorylation of neurofilament heavy chain (NF-H) increases neurofilament bundling and reduces their anterograde axonal transport [36].

\section{Mitogen-activated protein kinases (MAPKs)}

MAPKs are serine/threonine protein kinases that coordinate cellular responses to diverse extracellular stimuli. In its simplest form, MAPK signaling can be considered as a cascade of three tiers of protein kinases: MAPK kinase kinases (ASK1, TAK1, RAF1, MLK, MEKK4), MAPK kinases (MEK1, MKK4/7 and MKK3/6) and MAPKs (ERKs, JNKs and p38 MAPKs). However, representing the MAPK cascade as three isolated pathways is an 
oversimplification; the MAPK signaling network functionally interacts with many other pathways via shared scaffolding proteins, which target them to specific cellular locations [37]. In addition, there are several isoforms of each MAPK, each with distinct functions [37].

ERK1/2. Extracellular-signal-regulated kinases 1 and 2 (ERK1/2, also known as p44/42 MAPK) are activated in response to mitogens and growth factors, including neurotrophins [38], and have been shown to play a key role in neuronal differentiation, plasticity and survival [39, 40]. ERK1/2 directly phosphorylate both kinesin-1 (Figure 1) and dynein (Figure 2) and thereby regulate their association with cargoes [41, 42]. In rodent cortical neurons, ERK1/2-mediated phosphorylation of DIC1B and DIC2C increases the association of dynein with TrkB/Rab7-positive signaling endosomes, leading to an increase in the number of signaling endosomes moving in the retrograde direction (Figure 2) [42]. Interestingly, phosphorylation of DIC1B and DIC2C by ERK1/2 does not alter the ability of dynein to bind to mitochondria in these neurons, suggesting that mitochondria utilize a different mechanism to recruit dynein [42]. By contrast, ERK1/2-induced phosphorylation of KLC1 at Ser460 prevents binding of the motor to APP-labeled vesicles [41, 43], leading to a decrease in their anterograde transport frequency (Figure 1) [41]. ERK1/2mediated phosphorylation of KLC1, however, has no effect on the axonal transport of other cargoes, such as CRMP2 or the neurotrophin receptor scaffolding protein Kidins220/ARMS [41]. This suggests that ERK1/2 only mediate the fast retrograde axonal transport of specific cargoes.

Interestingly, activated ERK1/2 are also cargoes of signaling endosomes and are transported to the cell body where they induce changes in neurotrophindependent gene expression [44]. This raises the question of how the transport machinery is protected from spurious modification by transported kinases. Although the exact mechanism is currently unknown, it could be speculated that the interaction of ERK1/2 with vimentin, which prevents dephosphorylation and deactivation of the kinases [45], could also preclude ERK1/2 from phosphorylating the transport machinery en route. 
ERK1/2 have also been shown to facilitate the slow anterograde axonal transport of neurofilaments [36]. ERK1/2 attenuate GSK3 $\beta$-mediated bundling of neurofilaments, leading to an increase in their transported pool [36]. However, ERK1/2 activity has no effect on the slow axonal transport of tau, indicating this regulatory mechanism is also cargo-specific [46].

p38 MAPKs. p38 MAPKs are activated in response to pro-inflammatory cytokines, such as tumor necrosis factor $\alpha$ (TNFa) and interleukin (IL)-1 $\beta$, and cellular stresses, such as hypoxic, genotoxic or oxidative stress [47]. There are four p38 MAPK isoforms: $\alpha, \beta, y$ and $\delta$. p38 MAPK $\alpha$ and $\beta$ are considered to be the major isoforms expressed in the central nervous system [48].

p38 MAPK is a negative regulator of both fast and slow axonal transport. In squid axoplasm, p38 MAPKa directly phosphorylates KIF5C at Ser175/Ser176 and thereby inhibits the fast anterograde axonal transport of membrane-bound organelles (Table 1) [48]. In contrast, p38 MAPK $\beta$ inhibits both anterograde and retrograde axonal transport in this system [48]. p38 MAPK-mediated inhibition of fast axonal transport has also been described for mitochondria in rodent hippocampal neurons [48, 49] and synaptic vesicles in Drosophila segmental nerves [50] (Figure 1 and 2), suggesting that this regulatory mechanism is conserved between species. However, the targets of this kinase in the mammalian transport machinery are presently unknown.

Activation of p38 MAPK has also been shown to inhibit the slow anterograde axonal transport of neurofilaments [51]. p38 MAPKa phosphorylates the tail domains of neurofilament medium (NF-M) and NF-H chains and regulates their attachment to motor proteins [52]. In rat cortical neurons, increased phosphorylation of NF-M and NF-H slows their axonal transport and induces bundling, leading to large accumulations of neurofilaments in the cell body and proximal axons [53].

JNKs. The c-Jun N-terminal kinases (JNKs) are encoded by three genes: JNK1 and 2 are expressed ubiquitously, while JNK3 is only expressed in neurons [11]. Similarly to p38 MAPK, JNKs are activated by cellular stress 
and pro-inflammatory cytokines [54]. In addition to their physiological roles in neuronal development and synaptic plasticity, over-activation of JNKs has been implicated in several neurological disorders [11].

JNKs regulate axonal transport via direct phosphorylation of motors, adapter proteins and cargoes. In squid axoplasm, JNK3 inhibits fast anterograde axonal transport via direct phosphorylation of KIF5C at Ser176, reducing kinesin- 1 function and its ability to bind to microtubules [55, 56]. Activation of JNK also inhibits the axonal transport of synaptic vesicles and mitochondria in rodent hippocampal neurons (Figure 1 and 2) [57]. In these studies, JNK was found to directly phosphorylate KIF5B and induce its dissociation from microtubules [57]. The mechanism responsible for JNK-mediated inhibition of retrograde axonal transport remains unclear (Figure 2).

JNK also regulates fast axonal transport via phosphorylation of JNK interacting protein 1 (JIP1). JIPs not only act as signaling platforms for JNKs [58], but also serve as adapter proteins for both dynein and kinesin [59, 60] (Figure 1 and 2). In rodent neurons, phosphorylation of JIP1 at Ser421 stabilizes its interaction with kinesin-1 and promotes the anterograde axonal transport of APP, whilst expression of a non-phosphorylatable JIP1 mutant favors retrograde axonal transport [60]. Thus, JNK-mediated phosphorylation of JIP1 could act as a molecular switch between retrograde and anterograde axonal transport [60].

Similarly to ERK1/2, JNK is also a signaling endosome cargo and its retrograde axonal transport is essential for the response to axonal injury [61]. Given the inhibitory effect of JNK on retrograde axonal transport, there must be additional regulatory mechanisms in place to allow its processive transport along the axon. It has been suggested that JIP-1 modulates JNK signaling during transport via association with protein phosphatases [62]. For example, JIP1 has been shown to bind the dual-specificity phosphatase MAPK phosphatase 7 (MKP7), which dephosphorylates and inactivates JNK [63]. 
Finally, JNK1 has been shown to negatively regulate slow anterograde axonal transport. In chick sensory neurons, JNK1 was found to directly phosphorylate neurofilaments, leading to inhibition of their slow axonal transport [64].

\section{Cyclin Dependent Kinase 5 (Cdk5)}

Cdk5 was first discovered based on its sequence homology to other cyclindependent kinases such as Cdc2 and Cdk2 [65, 66]. However, unlike other cyclin-dependent kinases, Cdk5 does not play a direct role in cell cycle control. Instead, Cdk5 regulates several processes in the nervous system, including neurite outgrowth, synaptic activity and neuronal migration [12], and its deregulation has been linked to neurodegeneration $[67,68]$.

The role of Cdk5 in the regulation of axonal transport is controversial, with dispute over whether the kinase regulates axonal transport under normal conditions or only in response to cellular stress. In squid axoplasm and $C$. elegans motor neurons, inhibition of Cdk5 was reported to disrupt the anterograde axonal transport of membrane-bound organelles [69, 70] and the retrograde axonal transport of synaptic vesicles [71]. Similarly, expression of kinase-dead Cdk5 led to inhibition of both anterograde and retrograde axonal transport of acidic organelles in rodent DRGs [72]. Experiments in squid axoplasm suggest that Cdk5 regulates axonal transport indirectly via inhibition of GSK3 $\beta$ (see GSK3 $\beta$ section) [70]. Cdk5 has been shown to activate lemur tyrosine kinase-2 (LMTK2), which in turn phosphorylates and activates protein phosphatase 1 (PP1), thus preventing GSK3 $\beta$ activation [70, 73, 74]. Cdk5 has also been described to regulate fast retrograde axonal transport through phosphorylation of the dynein adapter protein Ndel1. In rodent DRGs, phosphorylation of Ndel1 by Cdk5 increases its association with Lis1, leading to enhanced retrograde axonal transport of acidic organelles (Figure 2) [72].

However, a more recent study performed in rodent DRGs has failed to reproduce these results. In this study, expression of kinase-dead Cdk5 did not affect the axonal transport of lysosomes, mitochondria or TrkB-containing endosomes [75], suggesting that Cdk5 does not play a role in the regulation of axonal transport under normal conditions. By contrast, increasing Cdk5 
activity disrupted the axonal transport of these cargoes [75] suggesting that Cdk5 modulates axonal transport only under cellular stress. Although the reason for these conflicting results is unclear, it may be related to differences in the protocol used for DRG isolation and maintenance [72, 75].

Cdk5 has also been shown to negatively regulate the slow anterograde axonal transport of neurofilaments [36, 46, 76, 77]. Phosphorylation of neurofilaments by Cdk5 is proposed to disrupt their anterograde axonal transport by promoting bundling [36, 78], reducing binding to kinesin [79] and inhibiting the activity of ERK1/2 through phosphorylation-mediated deactivation of its upstream activator MEK1 [46]. Cdk5 also disrupts the slow anterograde axonal transport of tau, although the mechanism responsible remains unclear [46].

Crucially, the level of crosstalk between different kinases known to phosphorylate neurofilament subunits remains to be addressed, together with the physiological implications of the resulting combinatorial phosphorylation patterns.

\section{Akt}

The serine/threonine kinase Akt (also known as protein kinase B) has been shown to regulate the directionality of fast axonal transport. Activation of Akt in rodent hippocampal neurons leads to the preferential anterograde axonal transport of BDNF-containing secretory vesicles, APP-containing vesicles and synaptic vesicles through phosphorylation of the adapter protein htt (Figure 1) [80]. Akt-mediated phosphorylation of htt at Ser421 increases the recruitment of kinesin-1 to vesicles and also increases the amount of kinesin heavy chain bound to microtubules [80]. Inhibition of Akt causes an increase in the rate of retrograde axonal transport of BDNF-containing vesicles (Figure 2) [80]. Akt can also inactivate GSK3 $\beta$ and thereby release the inhibitory effect of GSK3 $\beta$ on the fast axonal transport of mitochondria in hippocampal neurons [24, 81]. It will be interesting to determine whether these regulatory mechanisms are conserved across species and neuronal types. 
Additional kinases implicated in the regulation of axonal transport

There is a small body of evidence implicating other protein kinases in the regulation of axonal transport (Key Figure). In invertebrate models, both protein kinase $A$ (PKA) and casein kinase 2 (CK2) inhibit fast anterograde axonal transport via direct phosphorylation of kinesin and reduction of its affinity for vesicular cargoes $[19,82]$. In addition, CK2 has been shown to inhibit retrograde axonal transport via an unknown mechanism [82].

However, these findings have not been confirmed in mammalian neurons. In contrast to its effects in an invertebrate model [19], PKA has been shown to facilitate the fast axonal transport of mitochondria in rodent DRGs [83]. It is unclear whether this reflects a difference in regulatory mechanisms between species or between different cargoes (mitochondria versus vesicular cargoes).

Protein kinase $\mathrm{C}$ has been demonstrated to be a negative regulator of both retrograde [84] and anterograde [85] fast axonal transport, but further work is required to confirm the PKC isoform(s) responsible, their target(s) and the mechanism of action at the molecular level.

Finally, overexpression of PTEN-induced putative kinase 1 (PINK1) has been shown to inhibit the bidirectional axonal transport of mitochondria through phosphorylation of the adapter protein Miro (Figure 1 and 2), which targets it for degradation [86].

\section{Relevance to CNS function and disease}

The regulation of axonal transport by protein kinases is essential for neuronal function and development. This mechanism has been shown to play an essential role in regulating axon integrity during pathfinding [87], establishing neuronal polarity [88] and regulating synaptic formation and maintenance [89]. Protein kinases also play a key role in the regulation of growth factor and stress signaling [90, 91] and the supply and clearance of proteins, lipids and organelles to the distal axon and synapse [27, 49, 80]. It is therefore not 
surprising that the deregulation of axonal transport by protein kinases has been implicated in several nervous system disorders (Table 2).

Amyotrophic lateral sclerosis (ALS)

The early appearance of axonal transport deficits in ALS mouse models [13, 92] suggests that they play a key role in disease pathogenesis. p38 MAPK is over-activated in spinal motor neurons of an ALS mouse model (SOD1G93A) $[48,93]$ and is responsible for defects in fast anterograde axonal transport induced by ALS-associated SOD1 mutants [48]. In addition, deregulation of p38 MAPK and Cdk5 is linked to the hyperphosphorylation of neurofilaments and subsequent disruption of their slow axonal transport observed in ALS [51, 53, 93, 94].

Huntington's disease (HD)

Mutant huntingtin (polyQ-htt) stimulates JNK3-mediated phosphorylation of KIF5C and induces deficits in both anterograde and retrograde axonal transport in squid axoplasm [56]. Interestingly, Akt-mediated phosphorylation of polyQ-htt at Ser421 can restore fast axonal transport to wild type levels [95].

Alzheimer's disease (AD)

$A \beta$ is proposed to inhibit fast axonal transport through Akt inhibition [96] and activation of CK2 [82], GSK3 $\beta$ [29, 96, 97] and p38 MAPK [49]. ADassociated presenilin 1 mutations and hyperphosphorylated tau have also been shown to induce axonal transport defects through activation of GSK3 $\beta$ [25, 96, 98]. Correcting fast axonal transport defects by reducing GSK3 $\beta$ activity was found to be beneficial in $A D$ models [99, 100]. Deregulation of Cdk5 is also linked to defects in the slow axonal transport of neurofilaments in AD [77].

Spinal bulbar muscular atrophy (SBMA)

SBMA is caused by a CAG repeat expansion in the first exon of the androgen receptor (polyQ-AR). Perfusion of the polyQ-AR in squid axoplasm inhibits the fast axonal transport of membrane-bound organelles through stimulation of 
JNK3-mediated phosphorylation of the kinesin heavy chain [55]. However, the role of axonal transport deficits in SBMA is controversial, since alterations in axonal transport were not found in vivo in a mouse model of SBMA expressing endogenous levels of polyQ-AR [101].

\section{Charcot-Marie-Tooth type 2F (CMT2F)}

CMT2F-mutant HSPB1 has been shown to induce alterations in the slow anterograde axonal transport of neurofilaments [79]. Mutant HSPB1 activates Cdk5, leading to neurofilament bundling and reduced association with kinesin1.

Over-activation of protein kinases and the subsequent disruption of axonal transport are a common occurrence in neurodegenerative diseases [102]. However, the direct demonstration that kinase-induced disruptions in axonal transport trigger disease pathogenesis is still lacking. The identification of pharmacological inhibitors of key protein kinases, such as GSK3 $\beta$, JNK3 and p38 MAPK, able to restore their activity to basal levels and normalize axonal transport, will help answer this question and determine whether protein kinases represent effective therapeutic targets for neurodegenerative diseases.

\section{Concluding remarks and future perspectives}

The complex scenario emerging from these studies highlights the crucial role played by protein kinases in the modulation of axonal transport. Motors, adapters and cargoes are clearly key targets for differential regulation by phosphorylation; something we are only just beginning to understand. Many intriguing questions still remain to be answered (see Outstanding Questions Box).

Given the key role that axonal transport plays in neuronal function, it is not surprising that alterations in protein kinase activity disrupt the axonal transport of selected cargoes and may lead to the severe pathological features of many nervous system disorders. Protein kinases may therefore represent key 
therapeutic targets for several neurological diseases that currently lack effective therapies. However, targeting protein kinases in neurological diseases presents a number of challenges [103]. In particular, there are likely to be problems with off-target effects, as protein kinases are often involved in the regulation of many cellular processes and participate in multiple signaling pathways. Understanding how to control the phosphorylation of specific substrates will aid the design of novel kinase inhibitors with fewer off-target effects. 


\section{Box 1. The structure of kinesin-1 and dynein/dynactin motor complexes}

Kinesin-1. Structural studies have revealed that kinesin-1 is a rod-shaped protein, with globular heads connected by a stalk to a tail domain. The carboxy terminal domains of the two KIF5 subunits associate with two KLCs and mediate cargo binding. The amino terminal globular heads of KIF5 form the motor domains and contain ATP and microtubule binding sites. Kinesin-1 is able to take steps along the microtubule by alternately detaching and advancing each of its two globular heads [104]. High-resolution X-ray crystallography and cryo-EM microscopy of kinesin-1 in complex with tubulin in both ATP-bound and nucleotide-free states have provided insights into how the motor moves [104]. ATP binding to the "front" motor domain not only allows high affinity binding to microtubules, but also triggers conformational changes in the "rear" globular head that allows it to detach from microtubules and take a step. Steps are driven by conversion of the neck linker domain from a disordered state to a well-defined structure.

Cytoplasmic dynein/dynactin. Dynein is composed of two heavy chains (DHCs) and additional intermediate (DICs), light intermediate (DLICs) and light chains (DLCs). The carboxy terminal region of DHC consists of a ring of six putative AAA+ ATPase domains (AAA1-6). ATP binding to AAA1 lowers the affinity of the microtubule-binding domain for microtubules so that the DHC can detach and diffuse to its next binding site. ATP hydrolysis induces remodeling of the linker domain and allows the motor to complete the step [105]. The amino terminus of the DHC mediates dimerization and binding to DICs, DLICs and DLCs to form the cargo-binding domain. DICs contain the dynactin-binding region. Dynactin consists of eight core subunits: p150 Glued, dynamitin/p50, Arp1, Arp11, p62, p24, p25 and p27 [106]. A dimer of p150Glued forms a bridge between the dynein intermediate chain and microtubules that is believed to increase motor processivity. Dynamitin/p50 overexpression inhibits dynein-mediated axonal transport by causing the release of $p 150^{\text {Glued }}$ and p24, thus disrupting the dynein-dynactin complex [107]. BICD2 is required to stabilize the interaction between dynein and dynactin, allowing the processive movement of the complex [108]. 


\section{Glossary}

Cargo: Organelles, vesicles, RNA or proteins that are carried by molecular motor complexes along microtubules.

Microtubules: polymers of tubulin that extend throughout axons and dendrites and form the tracks along which molecular motors move. Microtubules are polarized in axons, conferring directionality to axonal transport.

Neurofilaments: a type of intermediate filament found specifically in neurons. Neurofilaments are composed of three major subunits: light (NF-L), medium (NF-M) and heavy (NF-H). They are thought to provide structural support for axons, regulate axon diameter and act as a scaffold for various axonal components.

Protein kinase: an ATP-dependent enzyme (phosphotransferase) that adds a phosphate group to its protein substrate. Phosphorylation typically causes a functional change in the substrate, such as altering its activity, cellular location or association with other proteins.

Squid axoplasm: extruded cytoplasm of the giant axons of the squid Loligo pealeii. Differential interference contrast (DIC) microscopy is used to observe the bidirectional movement of membrane-bound organelles in real time. Axoplasm can be perfused with recombinant proteins and small molecular inhibitors in order to determine their effects on axonal transport. 


\section{Figure legends}

Figure 1. Cargo-specific regulation of anterograde axonal transport by protein kinases.

Examples of cargoes whose anterograde axonal transport is regulated by phosphorylation. Protein kinases that have been shown to regulate the anterograde transport of specific cargoes are listed. Their molecular targets are indicated in parentheses. For many of the molecular targets, the phosphorylation site(s) have been identified (see Table 1). Question marks indicate that contradicting results have been reported on the effect of the specific protein kinase on axonal transport.

Figure 2. Cargo-specific regulation of retrograde axonal transport by protein kinases.

Examples of cargoes whose retrograde axonal transport is regulated by phosphorylation. Protein kinases that have been shown to regulate the retrograde transport of specific cargoes are listed, together with their molecular targets, if known. For many of the molecular targets, the phosphorylation site(s) have been identified (see Table 1). 
Table 1. Protein kinases that directly regulate axonal transport ${ }^{a}$

\begin{tabular}{|c|c|c|c|c|c|c|}
\hline \multirow{2}{*}{ Kinase } & \multirow{2}{*}{ Target } & \multirow{2}{*}{ Cargo affected } & \multicolumn{2}{|c|}{ Effect on AT } & \multirow{2}{*}{ Type of neuron } & \multirow{2}{*}{ Ref } \\
\hline & & & aAT & rAT & & \\
\hline \multirow[t]{5}{*}{ GSK3 $\beta$} & KLC2 & Membrane-bound organelles & - & - & Squid axoplasm & {$[23,26]$} \\
\hline & DIC1B (Ser87/Thr88); DIC2C (Ser88/Thr89) & Acidic organelles & NE & - & Dorsal root ganglia & {$[28]$} \\
\hline & $?$ & $\begin{array}{l}\text { Mitochondria, APP-containing vesicles, } \\
\text { lipid droplets, synaptic vesicles (?), } \\
\text { BDNF-containing secretory vesicles }\end{array}$ & - & - & $\begin{array}{l}\text { Hippocampal neurons, } \\
\text { Drosophila segmental nerves }\end{array}$ & {$[24,25,27,29]$} \\
\hline & Tau & Tau & + & & Cortical neurons & [35] \\
\hline & $\mathrm{NF}-\mathrm{H}$ & Neurofilaments & - & & NB2a/d1 cells & [36] \\
\hline \multirow[t]{3}{*}{ ERK1/2 } & DIC1B (Ser80); DIC2C (Ser81) & Signaling endosomes & & + & Hippocampal neurons & [42] \\
\hline & KLC1 (Ser460) & Calsyntenin-associated vesicles (APP) & - & + & Cortical neurons & [41] \\
\hline & GSK3ß (Ser9) & Neurofilaments & + & & NB2a/d1 cells, dorsal root ganglia & {$[36]$} \\
\hline \multirow[t]{4}{*}{ p38 MAPK } & KIF5C (Ser175/176) & Membrane-bound organelles & - & NE & Squid axoplasm & [48] \\
\hline & $?$ & Mitochondria & - & - & Hippocampal neurons & [49] \\
\hline & $?$ & Synaptic vesicles & - & & Drosophila segmental nerves & {$[50]$} \\
\hline & NF-M and $-\mathrm{H}$ & Neurofilaments & - & & Cortical neurons & {$[51,53]$} \\
\hline \multirow[t]{4}{*}{ JNK } & JIP1 (Ser421) & APP-containing vesicles & + & - & Dorsal root ganglia & {$[60]$} \\
\hline & KIF5C (Ser176) & Membrane-bound organelles & - & - & Squid axoplasm & {$[55,56]$} \\
\hline & KIF5B & Mitochondria, synaptic vesicles & - & - & Hippocampal neurons & {$[55-57]$} \\
\hline & $?$ & Neurofilaments & - & & NB2a/d1 cells, dorsal root ganglia & {$[64]$} \\
\hline \multirow[t]{5}{*}{ Cdk5 } & LMTK2/PP1 & Membrane-bound organelles & + & + & Squid axoplasm, dorsal root ganglia & {$[70,109]$} \\
\hline & $?$ & Synaptic vesicles & + & & C.elegans motor neurons & [110] \\
\hline & Ndel1 & Acidic organelles & + & + & Dorsal root ganglia & [72] \\
\hline & MEK & Neurofilaments & - & & NB2a/d1 cells & [46] \\
\hline & NF-M and $-\mathrm{H}$ & Neurofilaments & - & & Dorsal root ganglia, SH-SY5Y & {$[36,76,77,79]$} \\
\hline \multirow[t]{2}{*}{ Akt } & htt (Ser421) & $\begin{array}{l}\text { BDNF-containing secretory vesicles, } \\
\text { APP-containing vesicles, synaptic vesicles }\end{array}$ & + & - & Cortical neurons & [80] \\
\hline & GSK3ß (Ser9) & Mitochondria & + & + & Hippocampal neurons & {$[24,81]$} \\
\hline \multirow[t]{2}{*}{ PKA } & Kinesin & Small vesicles (not mitochondria) & - & NE & Crayfish walking leg giant axon & {$[19]$} \\
\hline & $?$ & Mitochondria & + & + & Dorsal root ganglia & [83] \\
\hline CK2 & KLC1 and 2 & Membrane-bound organelles & - & - & Squid axoplasm & [82] \\
\hline PKC & $?$ & Membrane-bound organelles & - & - & $\begin{array}{l}\text { Sympathetic and sensory neurons, } \\
\text { squid axoplasm }\end{array}$ & {$[84,85]$} \\
\hline PINK1 & Miro (Ser156) & Mitochondria & - & - & Cortical neurons & {$[86]$} \\
\hline
\end{tabular}

a. rAT, retrograde axonal transport. aAT, anterograde axonal transport. +, enhanced; -, inhibited; NE, no effect. 
Table 2. Protein kinases implicated in the deregulation of axonal transport in neurological diseases ${ }^{\text {a }}$

\begin{tabular}{|c|c|c|c|c|c|c|c|}
\hline \multirow{2}{*}{ Kinase } & \multirow{2}{*}{ Target } & \multirow{2}{*}{ Cargo affected } & \multicolumn{2}{|c|}{ Effect on AT } & \multirow{2}{*}{ Type of neuron } & \multirow{2}{*}{ Disease association } & \multirow{2}{*}{ Ref } \\
\hline & & & aAT & rAT & & & \\
\hline \multirow[t]{4}{*}{ p38 MAPK } & KIF5C (Ser175/176) & Membrane-bound organelles & - & NE & Squid axoplasm & Amyotrophic lateral sclerosis & [48] \\
\hline & $?$ & Signalling endosomes (CTB) & - & & Optic nerve & Glaucoma & [111] \\
\hline & $?$ & Mitochondria & - & - & Hippocampal neurons & Alzheimer's disease & [49] \\
\hline & $\mathrm{NF}-\mathrm{M}$ and $-\mathrm{H}$ & Neurofilaments & - & & Cortical and motor neurons & Amyotrophic lateral sclerosis & {$[51,53,93]$} \\
\hline Cdk5 & $\mathrm{NF}-\mathrm{M}$ and $-\mathrm{H}$ & Neurofilaments & - & & SH-SY5Y, N2A & $\begin{array}{l}\text { Charcot-Marie-Tooth type 2F, } \\
\text { Alzheimer's disease }\end{array}$ & {$[36,76,77,79]$} \\
\hline CK2 & $\mathrm{KLC} 1$ and 2 & Membrane-bound organelles & - & - & Squid axoplasm & Alzheimer's disease & [82] \\
\hline \multirow[t]{2}{*}{ GSK3 $\beta$} & $?$ & $\begin{array}{l}\text { Synaptic vesicles, mitochondria, } \\
\text { APP-containing vesicles, BDNF- } \\
\text { containing secretory vesicles }\end{array}$ & - & - & $\begin{array}{l}\text { Hippocampal neurons, Drosophila } \\
\text { segmental nerves, squid axoplasm }\end{array}$ & Alzheimer's disease & $\begin{array}{l}{[23,25,27,29,} \\
96-98]\end{array}$ \\
\hline & KLC & Mitochondria & - & - & Cortical neurons & Krabbe disease & [23] \\
\hline \multirow[t]{2}{*}{ JNK3 } & KIF5C (Ser176) & Membrane-bound organelles & - & - & Squid axoplasm & Huntington's disease & [56] \\
\hline & KIF5 & Membrane-bound organelles & - & - & Squid axoplasm & $\begin{array}{l}\text { Spinal bulbar muscular } \\
\text { atrophy }\end{array}$ & [55] \\
\hline PKC & $?$ & Membrane-bound organelles & - & NE & Squid axoplasm & $\begin{array}{l}\text { Neurotoxin-induced } \\
\text { Parkinson's disease }\end{array}$ & [85] \\
\hline
\end{tabular}

a. rAT, retrograde axonal transport. aAT, anterograde axonal transport. +, enhanced; -, inhibited; NE, no effect. 


\section{ACKNOWLEDGMENTS}

We thank the members of the Molecular Neuropathobiology Laboratory for constructive comments and Ceri Davies (GSK) for critical reading of the manuscript. This work was supported by the BBSRC (KG) and UCL (GS). LG is the Graham Watts Senior Research Fellow, supported by the Brain Research Trust.

\section{AUTHOR INFORMATION}

\section{Affiliations}

Sobell Department of Motor Neuroscience \& Movement Disorders, UCL Institute of Neurology, University College London, WC1N 3BG London, UK Katherine Gibbs, Linda Greensmith, Giampietro Schiavo

\section{Author contributions}

$K G$ and $G S$ conceived the layout of the review, $K G$ drafted the manuscript, and $K G$ and $G S$ assembled the figures. All authors amended the manuscript and approved the submitted version.

\section{Competing financial interests}

The authors declare no competing financial interests.

\section{Corresponding author}

Correspondence to: Prof. Giampietro Schiavo, Sobell Department of Motor Neuroscience \& Movement Disorders, UCL Institute of Neurology, University College London, Queen Square, WC1N 3BG London, UK Phone: ++44 20 3448 4334, Fax: ++44 207813 3107; e-mail: giampietro.schiavo@ucl.ac.uk 


\section{References}

1 Schmieg, N., et al. (2014) Signalling endosomes in axonal transport: travel updates on the molecular highway. Semin. Cell Dev. Biol. 27, 32-43

2 Matusica, D. and Coulson, E.J. (2014) Local versus long-range neurotrophin receptor signalling: endosomes are not just carriers for axonal transport. Semin. Cell Dev. Biol. 31, 57-63

3 Doron-Mandel, E., et al. (2015) Growth control mechanisms in neuronal regeneration. FEBS Lett. 589, 1669-1677

4 Rishal, I., et al. (2012) A motor-driven mechanism for cell-length sensing. Cell reports 1, 608-616

5 Millecamps, S. and Julien, J.P. (2013) Axonal transport deficits and neurodegenerative diseases. Nat. Rev. Neurosci. 14, 161-176

6 Maday, S., et al. (2014) Axonal transport: cargo-specific mechanisms of motility and regulation. Neuron $84,292-309$

7 Kumar, S., et al. (2000) Cytoplasmic dynein ATPase activity is regulated by dynactin-dependent phosphorylation. J. Biol. Chem. 275, 31798-31804

8 Mcllvain, J.M., Jr., et al. (1994) Regulation of kinesin activity by phosphorylation of kinesin-associated proteins. J. Biol. Chem. 269, 1917619182

9 Seira, O. and Del Rio, J.A. (2014) Glycogen synthase kinase 3 beta (GSK3beta) at the tip of neuronal development and regeneration. Mol. Neurobiol. 49, 931-944

10 Hetman, M. and Gozdz, A. (2004) Role of extracellular signal regulated kinases 1 and 2 in neuronal survival. Eur. J. Biochem. 271, 2050-2055

11 Coffey, E.T. (2014) Nuclear and cytosolic JNK signalling in neurons. Nat. Rev. Neurosci. 15, 285-299

12 Kawauchi, T. (2014) Cdk5 regulates multiple cellular events in neural development, function and disease. Dev. Growth Differ. 56, 335-348

13 Bilsland, L.G., et al. (2010) Deficits in axonal transport precede ALS symptoms in vivo. Proc. Natl. Acad. Sci. U. S. A. 107, 20523-20528

14 Misgeld, T., et al. (2007) Imaging axonal transport of mitochondria in vivo. Nature methods 4, 559-561

15 Hirokawa, N., et al. (2010) Molecular motors in neurons: transport mechanisms and roles in brain function, development, and disease. Neuron $68,610-638$ 
16 Vale, R.D. (2003) The molecular motor toolbox for intracellular transport. Cell 112, 467-480

17 Castle, M.J., et al. (2014) Long-distance axonal transport of AAV9 is driven by dynein and kinesin-2 and is trafficked in a highly motile Rab7-positive compartment. Mol. Ther. 22, 554-566

18 Brown, C.L., et al. (2005) Kinesin-2 is a motor for late endosomes and lysosomes. Traffic (Copenhagen, Denmark) 6, 1114-1124

19 Okada, Y., et al. (1995) The activation of protein kinase A pathway selectively inhibits anterograde axonal transport of vesicles but not mitochondria transport or retrograde transport in vivo. J. Neurosci. 15, 30533064

20 Lo, K.Y., et al. (2011) KIF1A is the primary anterograde motor protein required for the axonal transport of dense-core vesicles in cultured hippocampal neurons. Neurosci. Lett. 491, 168-173

21 Conde, C. and Caceres, A. (2009) Microtubule assembly, organization and dynamics in axons and dendrites. Nat. Rev. Neurosci. 10, 319-332

22 Embi, N., et al. (1980) Glycogen synthase kinase-3 from rabbit skeletal muscle. Separation from cyclic-AMP-dependent protein kinase and phosphorylase kinase. Eur. J. Biochem. 107, 519-527

23 Cantuti Castelvetri, L., et al. (2013) The sphingolipid psychosine inhibits fast axonal transport in Krabbe disease by activation of GSK3beta and deregulation of molecular motors. J. Neurosci. 33, 10048-10056

24 Chen, S., et al. (2007) Serotonin stimulates mitochondrial transport in hippocampal neurons. Mol. Cell. Neurosci. 36, 472-483

25 Dolma, K., et al. (2014) Presenilin influences glycogen synthase kinase-3 beta (GSK-3beta) for kinesin-1 and dynein function during axonal transport. Hum. Mol. Genet. 23, 1121-1133

26 Morfini, G., et al. (2002) Glycogen synthase kinase 3 phosphorylates kinesin light chains and negatively regulates kinesin-based motility. EMBO J. $21,281-293$

27 Weaver, C., et al. (2013) Endogenous GSK-3/shaggy regulates bidirectional axonal transport of the amyloid precursor protein. Traffic (Copenhagen, Denmark) 14, 295-308

28 Gao, F.J., et al. (2015) GSK-3beta phosphorylation of cytoplasmic dynein reduces Ndel1 binding to intermediate chains and alters dynein motility. Traffic (Copenhagen, Denmark) In press

29 Decker, H., et al. (2010) Amyloid-beta peptide oligomers disrupt axonal transport through an NMDA receptor-dependent mechanism that is mediated 
by glycogen synthase kinase 3beta in primary cultured hippocampal neurons. J. Neurosci. 30, 9166-9171

30 Pigino, G., et al. (2003) Alzheimer's presenilin 1 mutations impair kinesinbased axonal transport. J. Neurosci. 23, 4499-4508

31 McKenney, R.J., et al. (2010) LIS1 and NudE induce a persistent dynein force-producing state. Cell 141, 304-314

32 Kawano, Y., et al. (2005) CRMP-2 is involved in kinesin-1-dependent transport of the Sra-1/WAVE1 complex and axon formation. Mol. Cell. Biol. 25, 9920-9935

33 Arimura, N., et al. (2009) Anterograde transport of TrkB in axons is mediated by direct interaction with Slp1 and Rab27. Dev. Cell 16, 675-686

34 Lovestone, S., et al. (1994) Alzheimer's disease-like phosphorylation of the microtubule-associated protein tau by glycogen synthase kinase-3 in transfected mammalian cells. Curr. Biol. 4, 1077-1086

35 Cuchillo-lbanez, I., et al. (2008) Phosphorylation of tau regulates its axonal transport by controlling its binding to kinesin. FASEB J. 22, 3186-3195

36 Lee, S., et al. (2014) Divergent and convergent roles for kinases and phosphatases in neurofilament dynamics. J. Cell Sci. 127, 4064-4077

37 Shaul, Y.D. and Seger, R. (2007) The MEK/ERK cascade: from signaling specificity to diverse functions. Biochim. Biophys. Acta 1773, 1213-1226

38 Roskoski, R., Jr. (2012) ERK1/2 MAP kinases: structure, function, and regulation. Pharmacol. Res. 66, 105-143

39 Li, L., et al. (2014) lonizing radiation causes increased tau phosphorylation in primary neurons. J. Neurochem. 131, 86-93

40 Newbern, J.M., et al. (2011) Specific functions for ERK/MAPK signaling during PNS development. Neuron 69, 91-105

41 Vagnoni, A., et al. (2011) Phosphorylation of kinesin light chain 1 at serine 460 modulates binding and trafficking of calsyntenin-1. J. Cell Sci. 124, 10321042

42 Mitchell, D.J., et al. (2012) Trk activation of the ERK1/2 kinase pathway stimulates intermediate chain phosphorylation and recruits cytoplasmic dynein to signaling endosomes for retrograde axonal transport. J. Neurosci. 32, 15495-15510

43 Vagnoni, A., et al. (2012) Calsyntenin-1 mediates axonal transport of the amyloid precursor protein and regulates Abeta production. Hum. Mol. Genet. $21,2845-2854$ 
44 Heerssen, H.M., et al. (2004) Dynein motors transport activated Trks to promote survival of target-dependent neurons. Nat. Neurosci. 7, 596-604

45 Perlson, E., et al. (2006) Vimentin binding to phosphorylated Erk sterically hinders enzymatic dephosphorylation of the kinase. J. Mol. Biol. 364, 938-944

46 Moran, C.M., et al. (2005) Cdk5 inhibits anterograde axonal transport of neurofilaments but not that of tau by inhibition of mitogen-activated protein kinase activity. Brain Res. Mol. Brain Res. 134, 338-344

47 Plotnikov, A., et al. (2011) The MAPK cascades: signaling components, nuclear roles and mechanisms of nuclear translocation. Biochim. Biophys. Acta $1813,1619-1633$

48 Morfini, G.A., et al. (2013) Inhibition of fast axonal transport by pathogenic SOD1 involves activation of p38 MAP kinase. PLoS One 8, e65235

49 Guo, L., et al. (2013) Cyclophilin D deficiency rescues axonal mitochondrial transport in Alzheimer's neurons. PLoS One 8, e54914

50 Klinedinst, S., et al. (2013) Independent pathways downstream of the Wnd/DLK MAPKKK regulate synaptic structure, axonal transport, and injury signaling. J. Neurosci. 33, 12764-12778

51 Stevenson, A., et al. (2009) Riluzole protects against glutamate-induced slowing of neurofilament axonal transport. Neurosci. Lett. 454, 161-164

52 Miller, C.C., et al. (2002) Axonal transport of neurofilaments in normal and disease states. Cell. Mol. Life Sci. 59, 323-330

53 Ackerley, S., et al. (2004) p38alpha stress-activated protein kinase phosphorylates neurofilaments and is associated with neurofilament pathology in amyotrophic lateral sclerosis. Mol. Cell. Neurosci. 26, 354-364

54 Koch, P., et al. (2015) Inhibitors of c-Jun N-terminal kinases: an update. J. Med. Chem. 58, 72-95

55 Morfini, G., et al. (2006) JNK mediates pathogenic effects of polyglutamine-expanded androgen receptor on fast axonal transport. Nat. Neurosci. 9, 907-916

56 Morfini, G.a., et al. (2009) Pathogenic huntingtin inhibits fast axonal transport by activating JNK3 and phosphorylating kinesin. Nature Neuroscience 12, 864-871

57 Stagi, M., et al. (2006) Unloading kinesin transported cargoes from the tubulin track via the inflammatory c-Jun $\mathrm{N}$-terminal kinase pathway. FASEB $\mathrm{J}$. $20,2573-2575$

58 Whitmarsh, A.J. (2006) The JIP family of MAPK scaffold proteins. Biochem. Soc. Trans. 34, 828-832 
59 Chiba, K., et al. (2014) Quantitative analysis of APP axonal transport in neurons: role of JIP1 in enhanced APP anterograde transport. Mol. Biol. Cell $25,3569-3580$

$60 \mathrm{Fu}$, M.M. and Holzbaur, E.L. (2013) JIP1 regulates the directionality of APP axonal transport by coordinating kinesin and dynein motors. J. Cell Biol. 202, 495-508

61 Lindwall, C. and Kanje, M. (2005) Retrograde axonal transport of JNK signaling molecules influence injury induced nuclear changes in p-c-Jun and ATF3 in adult rat sensory neurons. Mol. Cell. Neurosci. 29, 269-282

62 Koushika, S.P. (2008) "JIP"ing along the axon: the complex roles of JIPs in axonal transport. Bioessays 30, 10-14

63 Willoughby, E.A., et al. (2003) The JNK-interacting protein-1 scaffold protein targets MAPK phosphatase-7 to dephosphorylate JNK. J. Biol. Chem. 278, 10731-10736

64 DeFuria, J. and Shea, T.B. (2007) Arsenic inhibits neurofilament transport and induces perikaryal accumulation of phosphorylated neurofilaments: roles of JNK and GSK-3beta. Brain Res. 1181, 74-82

65 Lew, J., et al. (1992) Purification and characterization of a novel prolinedirected protein kinase from bovine brain. J. Biol. Chem. 267, 13383-13390

66 Meyerson, M., et al. (1992) A family of human cdc2-related protein kinases. EMBO J.11, 2909-2917

67 Miller, N., et al. (2015) Non-aggregating tau phosphorylation by cyclindependent kinase 5 contributes to motor neuron degeneration in spinal muscular atrophy. J. Neurosci. 35, 6038-6050

68 Castro-Alvarez, J.F., et al. (2015) Cyclin-Dependent kinase 5 targeting prevents $\beta$-Amyloid aggregation involving glycogen synthase kinase $3 \beta$ and phosphatases. J. Neurosci. Res. 93, 1258-1266

69 Ratner, N., et al. (1998) A role for cyclin-dependent kinase(s) in the modulation of fast anterograde axonal transport: effects defined by olomoucine and the APC tumor suppressor protein. J. Neurosci. 18, 77177726

70 Morfini, G., et al. (2004) A novel CDK5-dependent pathway for regulating GSK3 activity and kinesin-driven motility in neurons. EMBO J. 23, 2235-2245

71 Ou, C.Y., et al. (2010) Two cyclin-dependent kinase pathways are essential for polarized trafficking of presynaptic components. Cell 141, 846858

72 Pandey, J.P. and Smith, D.S. (2011) A Cdk5-dependent switch regulates Lis1/Ndel1/dynein-driven organelle transport in adult axons. J. Neurosci. 31, 17207-17219 
73 Manser, C., et al. (2012) Lemur tyrosine kinase-2 signalling regulates

79 Holmgren, A., et al. (2013) Charcot-Marie-Tooth causing HSPB1 mutations increase Cdk5-mediated phosphorylation of neurofilaments. Acta Neuropathol. 126, 93-108

80 Colin, E., et al. (2008) Huntingtin phosphorylation acts as a molecular switch for anterograde/retrograde transport in neurons. EMBO J. 27, 21242134

81 Chen, S., et al. (2008) Dopamine inhibits mitochondrial motility in hippocampal neurons. PLoS One 3, 1-15

82 Pigino, G., et al. (2009) Disruption of fast axonal transport is a pathogenic mechanism for intraneuronal amyloid beta. Proc. Natl. Acad. Sci. U. S. A. 106, 5907-5912

83 Hiruma, H., et al. (2002) Neuropeptide $Y$ inhibits axonal transport of particles in neurites of cultured adult mouse dorsal root ganglion cells. $J$. Physiol. 543, 85-97

84 Ozsarac, N., et al. (2003) Activation of protein kinase C inhibits retrograde transport of neurotrophins in mice. J. Neurosci. Res. 72, 203-210

85 Morfini, G., et al. (2007) 1-Methyl-4-phenylpyridinium affects fast axonal transport by activation of caspase and protein kinase C. Proc. Natl. Acad. Sci. U. S. A. $104,2442-2447$

86 Wang, X., et al. (2011) PINK1 and Parkin target Miro for phosphorylation and degradation to arrest mitochondrial motility. Cell 147, 893-906 
87 Shea, T.B. and Lee, S. (2011) Neurofilament phosphorylation regulates

89 Chua, J.J., et al. (2012) Phosphorylation-regulated axonal dependent transport of syntaxin 1 is mediated by a Kinesin-1 adapter. Proc. Natl. Acad. Sci. U. S. A. $109,5862-5867$

90 Harrington, A.W. and Ginty, D.D. (2013) Long-distance retrograde neurotrophic factor signalling in neurons. Nat. Rev. Neurosci. 14, 177-187

91 Perlson, E., et al. (2011) Retrograde Axonal Transport: Pathways To Cell Death? Trends Neurosci. 33, 335-344

92 Williamson, T.L. and Cleveland, D.W. (1999) Slowing of axonal transport is a very early event in the toxicity of ALS-linked SOD1 mutants to motor neurons. Nat. Neurosci. 2, 50-56

93 Tortarolo, M., et al. (2003) Persistent activation of p38 mitogen-activated protein kinase in a mouse model of familial amyotrophic lateral sclerosis correlates with disease progression. Mol. Cell. Neurosci. 23, 180-192

94 Nguyen, M.D., et al. (2001) Deregulation of Cdk5 in a mouse model of ALS: toxicity alleviated by perikaryal neurofilament inclusions. Neuron 30 , $135-147$

95 Zala, D., et al. (2008) Phosphorylation of mutant huntingtin at S421 restores anterograde and retrograde transport in neurons. Hum. Mol. Genet. $17,3837-3846$

96 Takach, O., et al. (2015) Modulation of insulin signaling rescues BDNF transport defects independent of tau in amyloid-beta oligomer-treated hippocampal neurons. Neurobiol. Aging 36, 1378-1382

97 Ramser, E.M., et al. (2013) Amyloid-beta oligomers induce tauindependent disruption of BDNF axonal transport via calcineurin activation in cultured hippocampal neurons. Mol. Biol. Cell 24, 2494-2505

98 Kanaan, N.M., et al. (2011) Pathogenic forms of tau inhibit kinesindependent axonal transport through a mechanism involving activation of axonal phosphotransferases. J. Neurosci. 31, 9858-9868

99 Shaw, J.L. and Chang, K.T. (2013) Nebula/DSCR1 upregulation delays neurodegeneration and protects against APP-induced axonal transport defects by restoring calcineurin and GSK-3beta signaling. PLoS Genet 9, e1003792 
100 Vossel, K.A., et al. (2015) Tau reduction prevents Abeta-induced axonal transport deficits by blocking activation of GSK3beta. J. Cell Biol. 209, 419433

101 Malik, B., et al. (2011) Absence of disturbed axonal transport in spinal and bulbar muscular atrophy. Hum. Mol. Genet. 20, 1776-1786

102 Kanaan, N.M., et al. (2013) Axonal degeneration in Alzheimer's disease: when signaling abnormalities meet the axonal transport system. Exp. Neurol. 246, 44-53

103 Chico, L.K., et al. (2009) Targeting protein kinases in central nervous system disorders. Nature reviews. Drug discovery 8, 892-909

104 Wang, W., et al. (2015) Kinesin, 30 years later: Recent insights from structural studies. Protein Sci. 24, 1047-1056

105 Carter, A.P. (2013) Crystal clear insights into how the dynein motor moves. J. Cell Sci. 126, 705-713

106 Urnavicius, L., et al. (2015) The structure of the dynactin complex and its interaction with dynein. Science 347, 1441-1446

107 Melkonian, K.A., et al. (2007) Mechanism of dynamitin-mediated disruption of dynactin. J. Biol. Chem. 282, 19355-19364

108 McKenney, R.J., et al. (2014) Activation of cytoplasmic dynein motility by dynactin-cargo adapter complexes. Science 345, 337-341

$109 \mathrm{Li}$, C., et al. (2004) Correlation between semaphorin3A-induced facilitation of axonal transport and local activation of a translation initiation factor eukaryotic translation initiation factor 4E. J. Neurosci. 24, 6161-6170

110 Goodwin, P.R., et al. (2012) Cyclin-dependent kinase 5 regulates the polarized trafficking of neuropeptide-containing dense-core vesicles in Caenorhabditis elegans motor neurons. J. Neurosci. 32, 8158-8172

111 Dapper, J.D., et al. (2013) Proximal inhibition of p38 MAPK stress signaling prevents distal axonopathy. Neurobiol. Dis. 59, 26-37 


\section{Outstanding Questions}

- Do kinases other than ERK1/2 and GSK3 $\beta$ directly phosphorylate cytoplasmic dynein? What effects does this process have on axonal transport?

- Do protein kinases phosphorylate the dynactin complex and, if so, how does this affect the activity and processivity of the dynein motor?

- Are kinesin-2 and -3 regulated by direct phosphorylation as seen for kinesin-1?

- Is the axonal transport of RNA also regulated by protein kinases? Which aspects of the regulatory network are shared between the different classes of organelles and how is specificity achieved?

- What are the molecular mechanisms underlying the deregulation of protein kinases in neurodegenerative diseases and how is axonal transport affected?

- Can we specifically target protein kinases and axonal transport as a therapeutic strategy for neurodegenerative diseases and other disorders of the nervous system? 
Marked-up manuscript - Combined comments

Click here to download Marked-up manuscript - Editor's comments: 2015 Gibbs TIBS Rev.docx $(2+2$

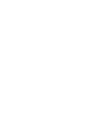
$\sqrt{10}$ $\sqrt{20}$ $\sqrt{20}$ .

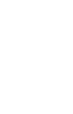
更 更 更 更 更 更 更 更 更 更 更 更 更 更 更 更 更 更 更 更 更

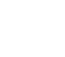
更 更 更 


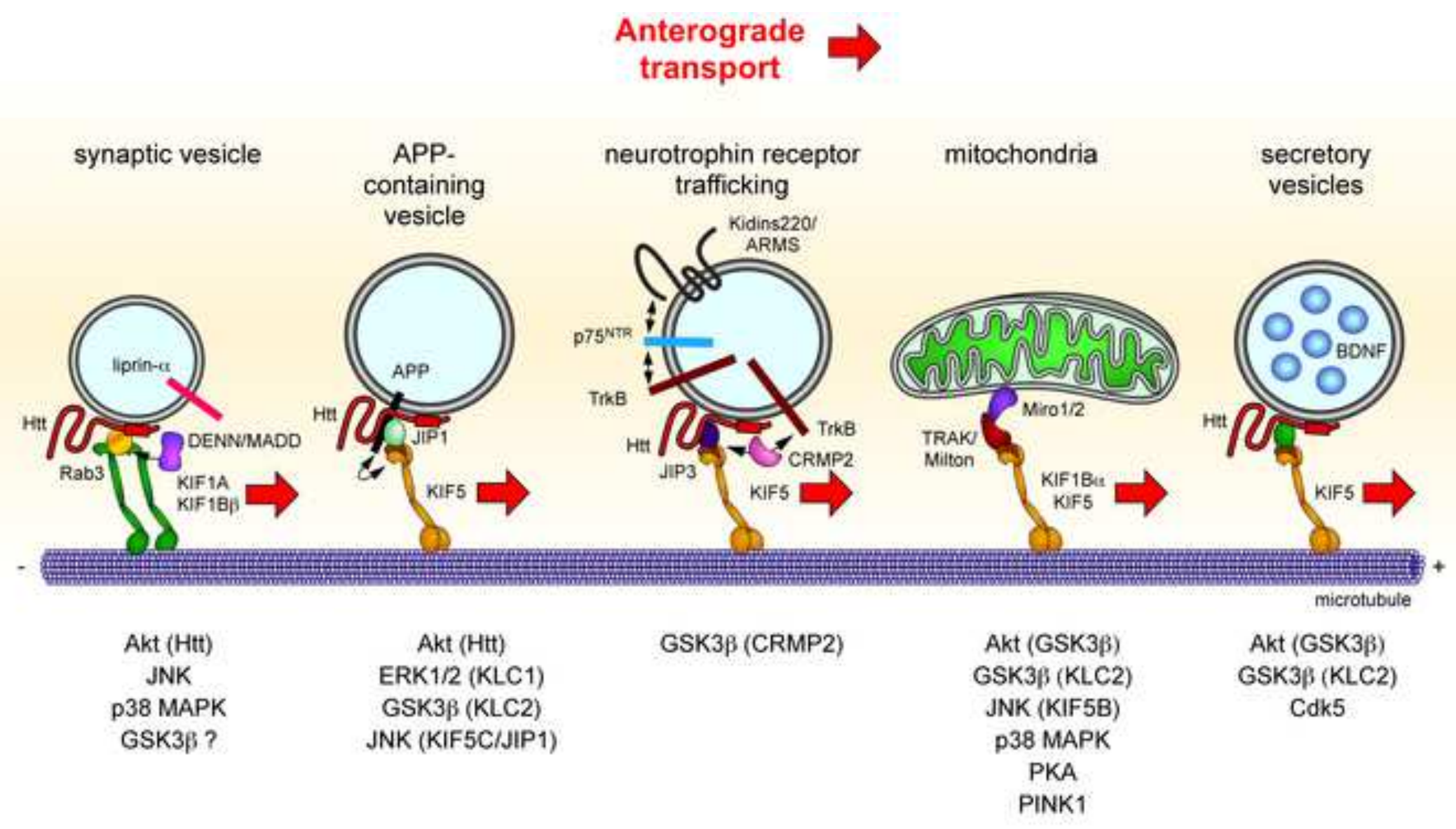




\section{1- $\begin{gathered}\text { Retrograde } \\ \text { transport }\end{gathered}$}

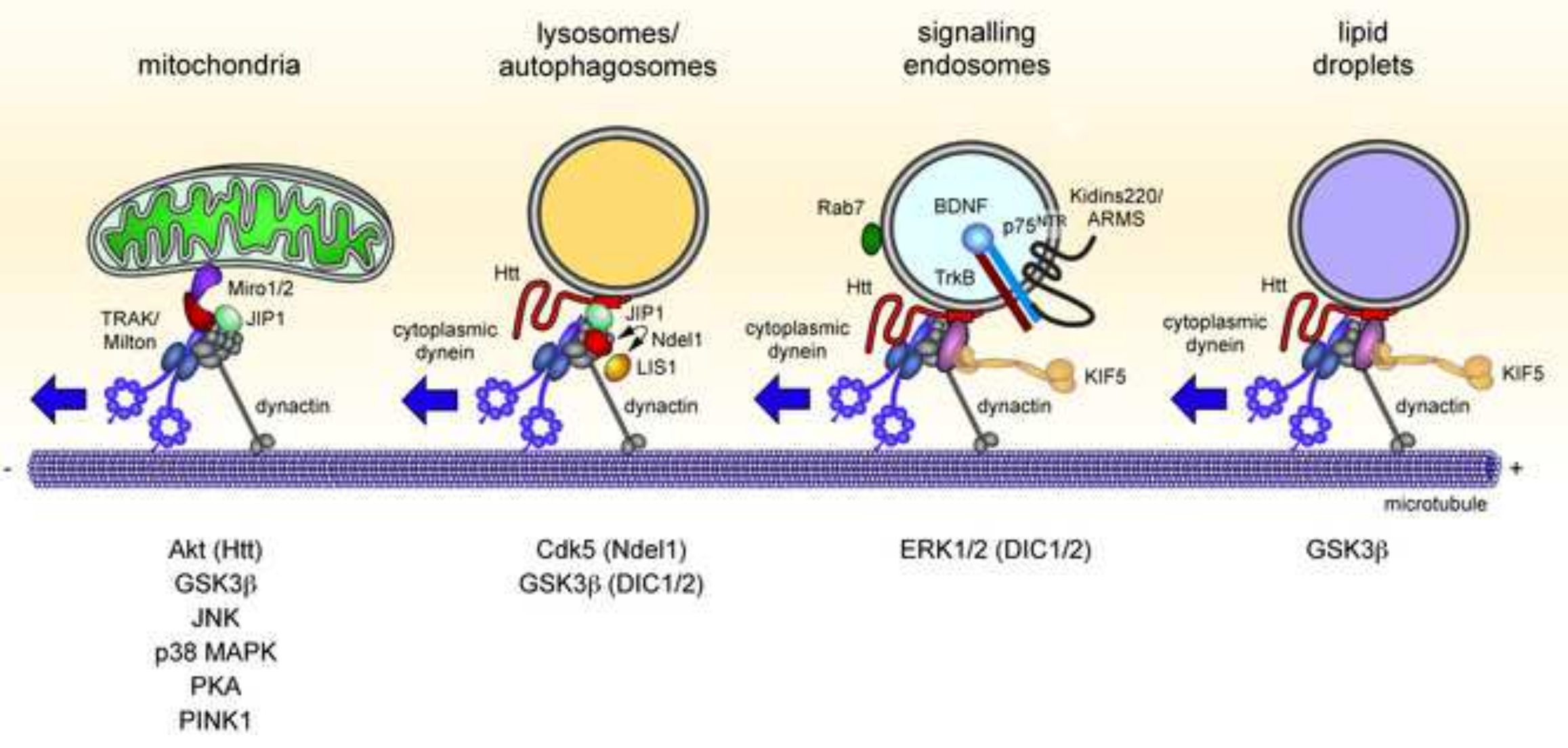




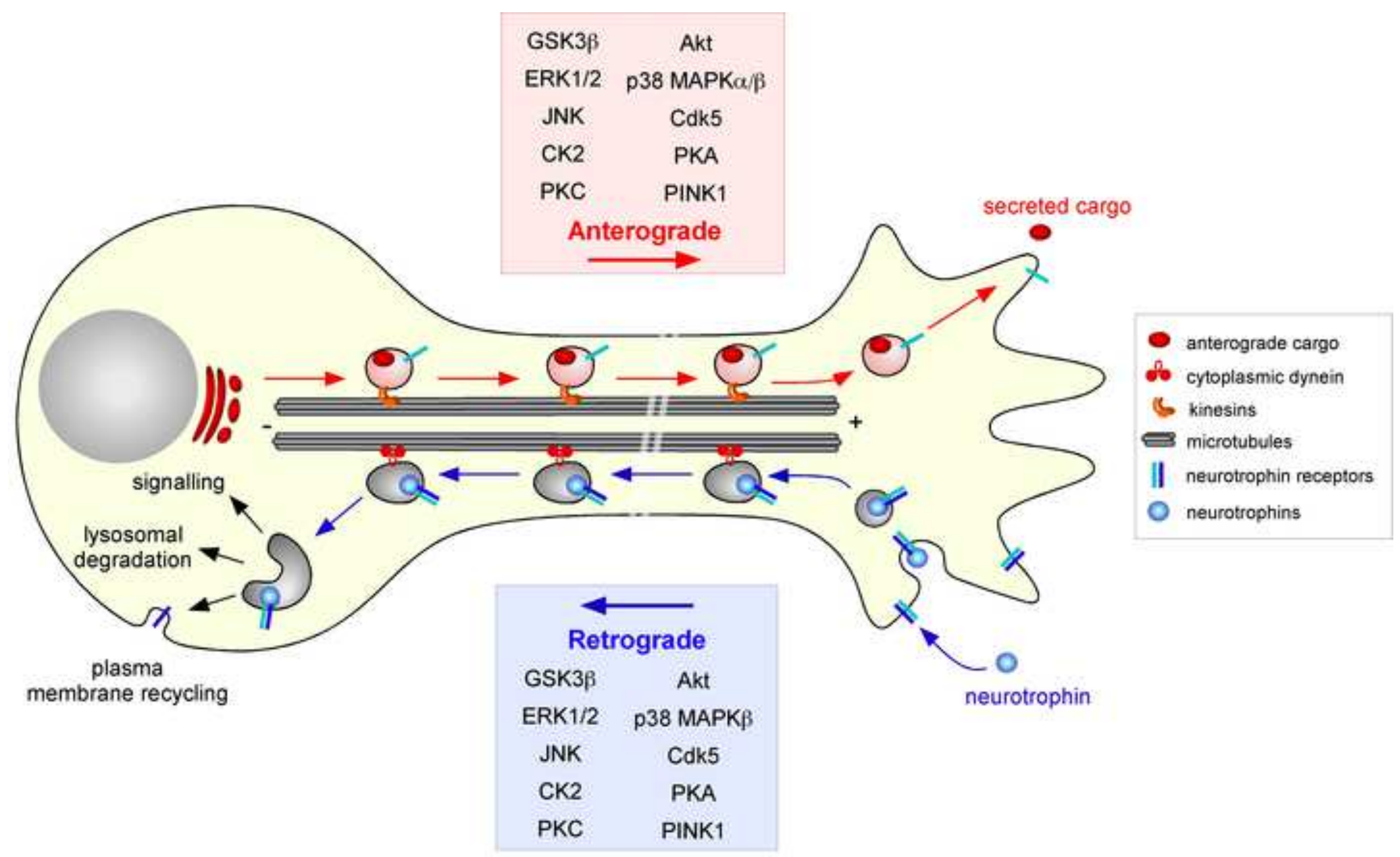


Original Figure 1 File
Click here to downloa

Original Figure 1 File
Click here to download Original Figure File: Gibbs Fig1.cvx

Click here to download Original Figl

riginal Figure File: Gibbs Fig1.cvx

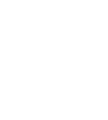

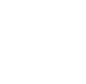
(n) (1)

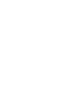

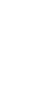
(1)

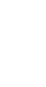

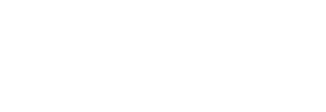

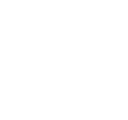
(1) (1) (1) (n)

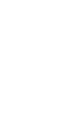

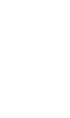
(1)

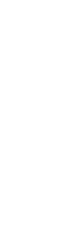

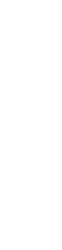

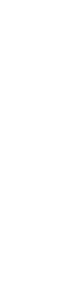


Original Figure 2 File
Click here to downloa

Original Figure 2 File
Click here to download Original Figure File: Gibbs Fig2.cvx

Click here to download Original Figure File: Gibbs Fig2.cvi

Figure File: Gibbs Fig2.cvx

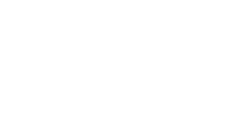
$x^{2}$

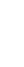

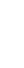

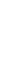
$x^{2}$

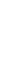

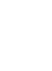

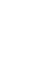

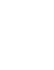

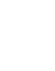

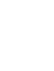

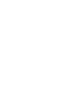

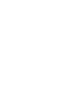

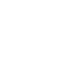
(1)

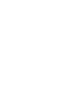
$x^{2}$ $x^{2}$ $x^{2}$ (1) $x^{2}$ $x^{2}$ (1) 
Original Key Figure File
Click here to download

Original Key Figure File
Click here to download Original Figure File: Glbbs key figure.cvx

Click here to download Original Figure File: Glb ks key figure.cv)

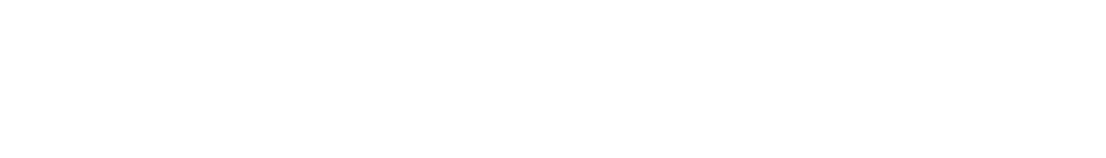

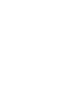
(1) (1) (1) (1) (1) (1) (1) (1) . . . . . . . .

(1)

(1) 
Original Figure 3 in Canvas $\mathrm{X}$ format
Click here to download Original Figure File: Gibbs3.cvx

Click here to download Original Figure File: Gibbs $3 . c \mathrm{x}$

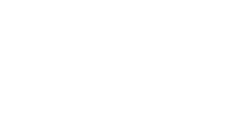

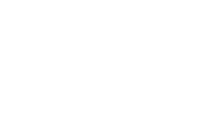

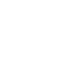

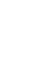

$\sqrt{2}$ (1) (1) (1) (1) (1) (1) (1) (1)

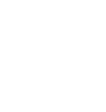
.

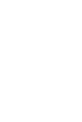

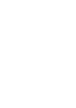

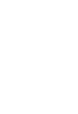

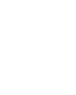

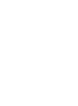
.

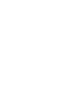
.

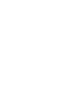
.

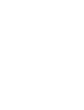

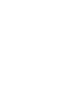

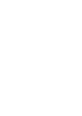
.

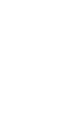

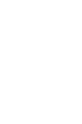
.

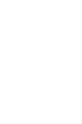
.

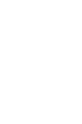
.

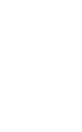
.

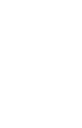
(

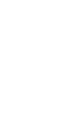
.

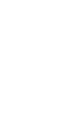
.

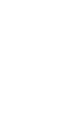
.

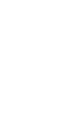
. . . .
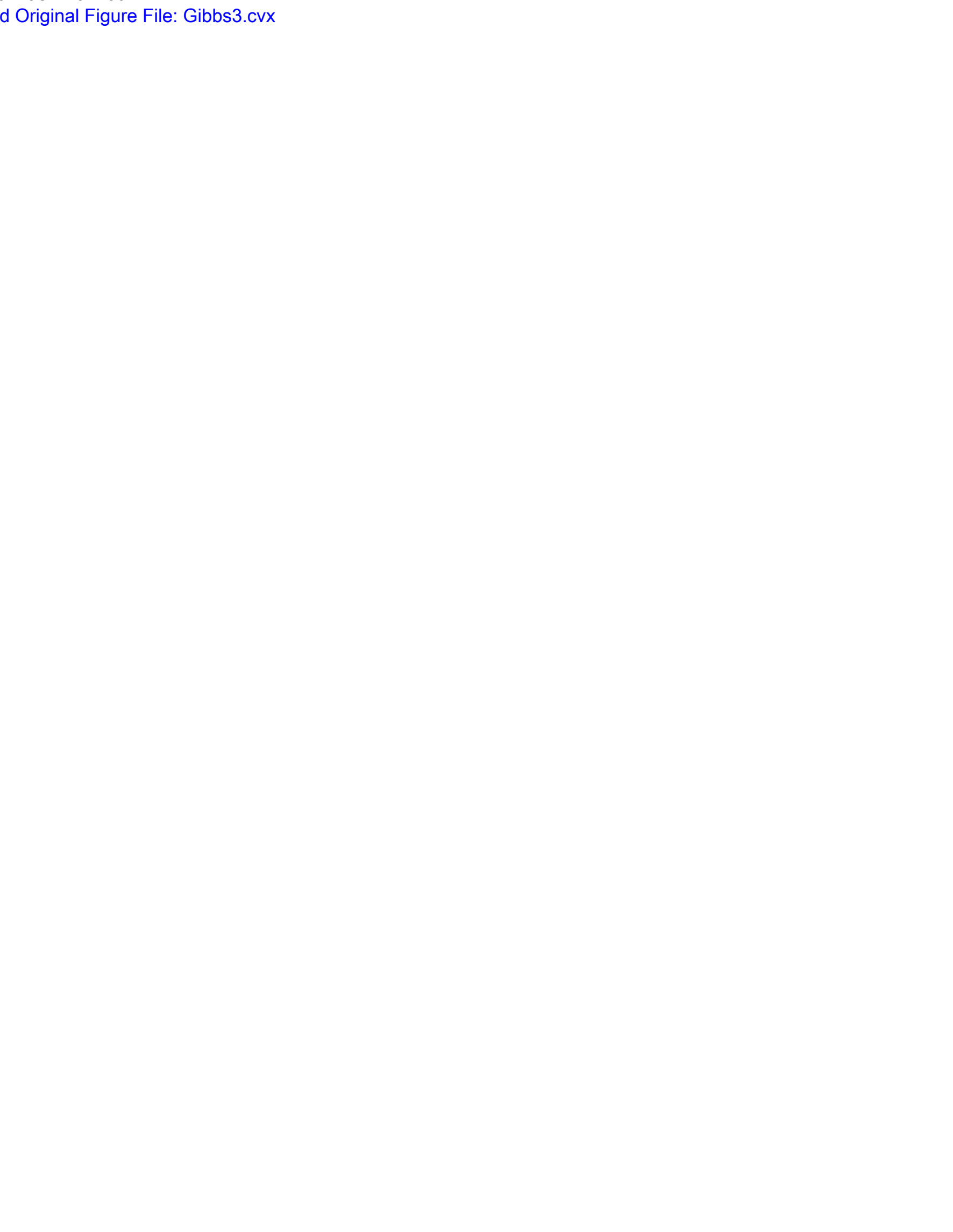